\title{
Smart Dropbox with Flexitime
}

\author{
P.Prashanti ${ }^{1}$, Tejaswini Roopali Patil ${ }^{2}$, Geethika Naraveni $^{3}$, Soumya Jangili ${ }^{4}$ \\ ${ }^{1}$ Assistant Professor- Dept of Electronics and Communication Engineering, BVRIT HYDERABAD College of \\ Engineering for Women, Hyderabad, India \\ ${ }^{2,3,4}$ UG Students- Dept of Electronics and Communication Engineering, BVRIT HYDERABAD College of Engineering for \\ Women, Hyderabad, India
}

\begin{abstract}
Most e-commerce websites face major issues when the house is locked or if the customer is unable to pick up the packages. Our solution includes a tie up with the e commerce websites and the customers. The delivery boy first undergoes an intense process of authentication via a validating key. After which he deposits the package in the container. Consecutively the customer is notified the location and time frame details as to when he/she can pick up the parcel at their convenience after a series of authentication protocol which includes a validation key. The entire process can be tracked by an app that connects the e-commerce website, the delivery boy and the consumer. The proposed system can be effectively built at a low cost with the integration of both hardware and software tools.
\end{abstract}

Keywords-E-commerce deliveries, raspberry pi, relay, electronic lock, Android studio.

\section{INTRODUCTION}

Nowadays the e-commerce markets have grown exponentially. Their basic concept is when a customer purchases an item the package will be delivered to the customers address. Due to the busy lifestyle of the people the ecommerce industry is facing major problems in delivering the packages to the customers safely [9]. One of their main concerns is what if at the time of parcel delivery if the house is locked. Then the delivery attempts to deliver the package later or the customer might ask for delivery at neighbors' location. Alternatively, the customer might ask to leave with security or at a personal mailbox or the final distribution centers agree to hold the package for an additional cost. These solutions are not effective and are time sensitive. Hence, we propose Smart Drop Box with flexi time as a solution. The customer can choose preferred locations for delivery. The delivery boy delivers the package to the chosen location and places it in a locker. The customer gets an OTP in the notifications to open the locker. The customer enters the OTP and can collect his package round the clock. Initially we assume that the locker is in open state. When the locker door is pushed forcefully the locker locks itself. When an order is to be delivered to a smart drop box location the delivery boy places the package into the locker and closes the door. Then in the android app that is downloaded he enters the locker number tracking number and the email address of the customer in separate fields that are provided in the android application. Then the delivery boy presses a lock button available. This will ensure that the data entered is saved in the database and a onetime password is generated which is mailed to the customer. Once the customer receives the pass word he can retrieve his parcel anytime by entering the OTP on the keypad that is attached to the drop box.

\section{II.WORKING AND IMPLEMENTATION}

The following are the steps that are to be followed for the working and implementation of our proposed device:

When a customer is shopping online on an e-commerce website, he has to select the preferred drop box location for delivery.

When the delivery man reaches the drop box location, he has to put the parcel into the drop box and shut it close physically. Now he has to open the smart drop box app and fill in the details of the customer such as tracking number, email Id, and drop box number. As soon as the details are entered and the lock button is pressed the details are saved into the database and an email is triggered containing the secret OTP. Once the customer receives the email, he is free to get back his package anytime he wants by entering the OTP onto the keypad placed on the drop box. Internally when the keypad is used to enter the correct OTP the raspberry pi and the relay decide the status of the lock i.e. if the electronic lock should be opened or remain closed.

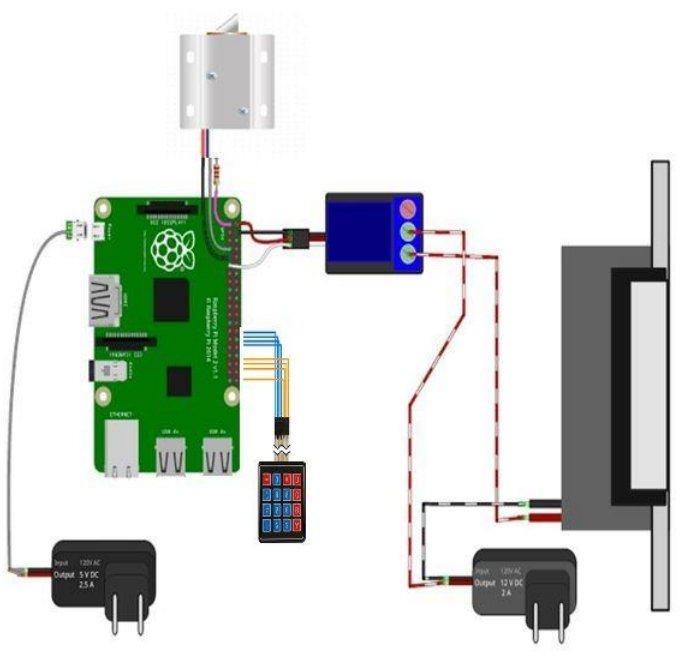

Fig. 2.1: Circuit Diagram 


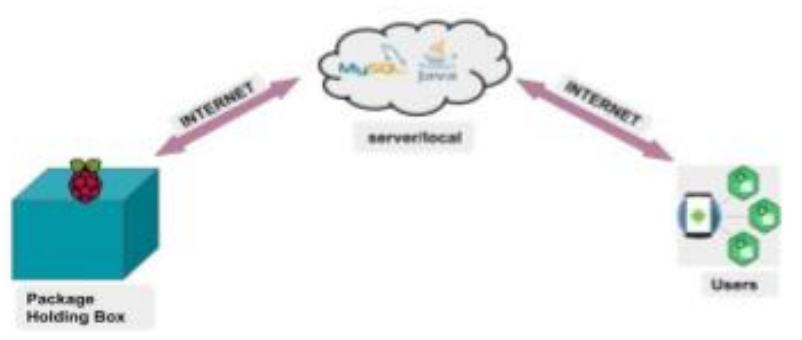

Fig 2.2: The Architecture of the Smart Dropbox with Flextime

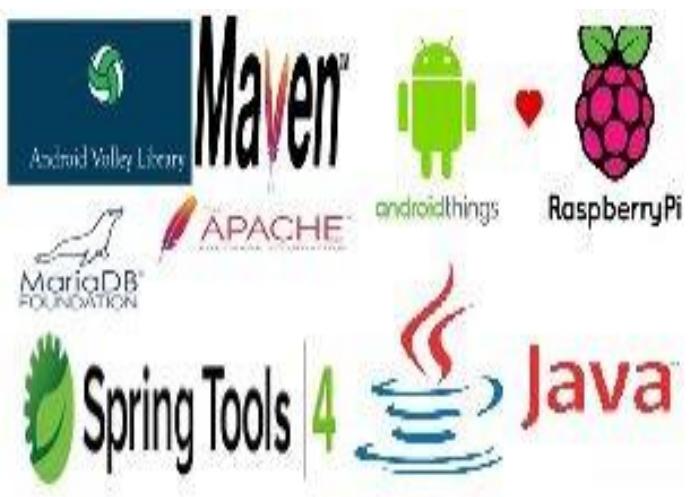

Fig. 2.3: Softwares used in building the smart drop box.

\section{III.RESULT}

The developed smart drop box is tested for all the functions and observed that the system works very accurately, only the authorized person can get the access to retrieve the package [5][6]. The system can be used by any user even though he doesn't know complete information about it.

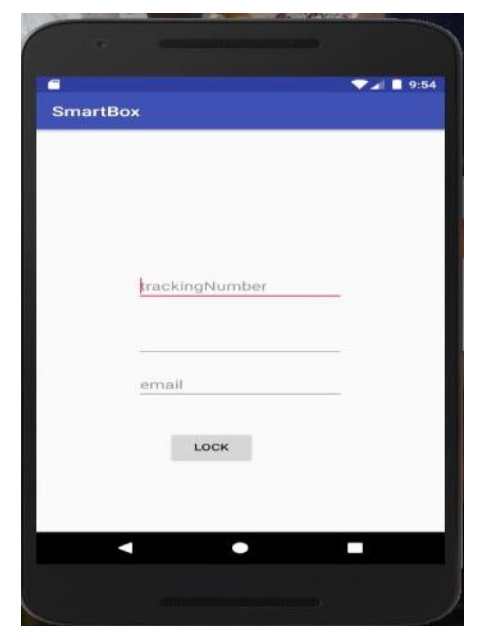

Fig.4 Smart Dropbox app installed and used by the delivery boy to insert data.
Once the lock button is pressed in the details are stored in the MySQL database and an email is triggered [2] .

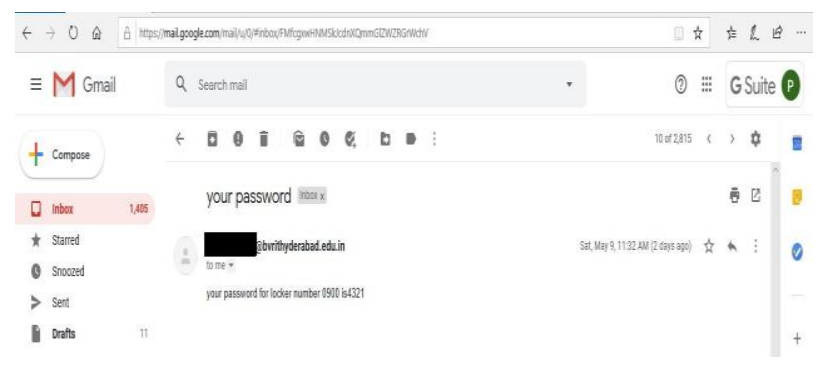

Fig. 3.2: Output of an email received by the customer containing OTP

A sample image of the wooden box that is installed at various locations is shown below.

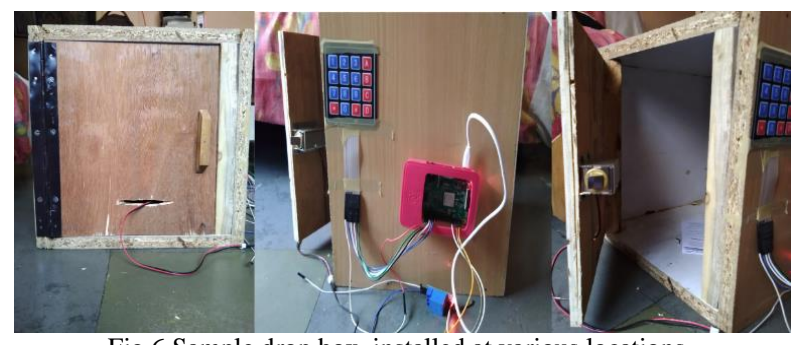

Fig.6 Sample drop box installed at various locations

\section{CONCLUSION}

An effective solution has been implemented for the troubles faced by the e-commerce websites in delivering the packages to locked houses or remote locations. This solution has proven to be both secure and cost effective. With high future scope various problem specific applications can be implemented with minor changes depending on the application.

\section{REFERENCES}

[1] http://history-computer.com/ModernComputer/Basis/relay.html

[2] http://www.mysql.com/customers/view/?id=720

[3] http://www.mysql.com/support/supportedplatforms/database.html

[4] https://docs.python.org/faq/general.html\#why-was-pythoncreated-in-the-first-place

[5] https://www.247asaplocksmith.com/encyclopedia/electronicdoor-locks

[6] http://aegispower.com/index.php/blog/192-cooling-methods-forpower-supplies

[7] https://www.raspberrypi.org/products/raspberry-pi-3-model-a-plus

[8] https://betanews.com/2017/07/19/raspberry-pi-eben-upton-qa/

[9] https://www.internet-of-strategy.com/the-importance-of-gooddelivery-for-e-commerce-businesses/ 